\title{
AVALIAÇÃO DA QUALIDADE FÍSICO-QUÍMICA DE COMPRIMIDOS DE ENALAPRIL: REFERÊNCIA, GENÉRICO E SIMILAR, DISPENSADOS NA REDE PÚBLICA E PRIVADA NA CIDADE DE MONTES CLAROS -MG
}

\author{
Rayssa Pinheiro Câmara \\ Discente do curso de Farmácia das Faculdades Integradas Pitágoras de Montes Claros-FIPMoc \\ Thiago Natan Viana Batista \\ Discente do curso de Farmácia das Faculdades Integradas Pitágoras de Montes Claros-FIPMoc \\ Leonardo Lanna \\ Docente do curso de Farmácia das Faculdades Integradas Pitágoras de Montes Claros-FIPMoc \\ Vanessa de Andrade Royo \\ Docente do curso de Farmácia das Faculdades Integradas Pitágoras de Montes Claros-FIPMoc \\ Docente do curso de Ciências Biológicas da UNIMONTES
}

Recebido em: 22/11/2013

Aprovado em: 24/03/2014

\section{RESUMO}

A hipertensão arterial (HA) é uma doença crônica e multifatorial. O enalapril é uma pró-droga inibidora da enzima conversora de angiotensina amplamente utilizado no tratamento da HA. Casos de inconformidades de medicamentos contendo enalapril foram relatados e representam um perigo para a saúde dos pacientes que deles necessitam. O objetivo deste estudo foi avaliar a qualidade dos medicamentos referência, genéricos, similares dispensados pela farmácia básica contendo enalapril. Para tanto, foi realizado o controle de qualidade físico-químico de quatro amostras de enalapril em comprimidos dispensados por farmácia básica e vendidos em farmácias localizadas em Montes Claros, MG, Brasil. As amostras foram avaliadas com relação ao aspecto visual, peso médio, resistência (dureza) e desintegração de acordo com as metodologias descritas na Farmacopeia Brasileira. Os resultados mostraram que todos os produtos avaliados foram aprovados em todos os testes. Assim, as amostras analisadas estavam em conformidade com as especificações oficiais.

Palavras-chave: Enalapril. Controle de qualidade. Comprimidos.

\section{QUALITY ASSESSMENT OF PILLS ENALAPRIL: REFERENCE, GENERIC AND SIMILAR, EXEMPT THE PUBLIC AND PRIVATE IN THE CITY OF MONTES CLAROS - MG}

\begin{abstract}
Arterial hypertension ( $\mathrm{AH})$ is a chronic multifactorial disease. Enalapril is a prodrug inhibiting angiotensin converting enzyme widely used in the treatment of $\mathrm{AH}$. Cases of noncompliance drug containing enalapril were reported and represent a danger to the health of patients who need them. The aim of this study was to evaluate the quality of the reference
\end{abstract}


CÂMARA, R. P.; BATISTA, T. N. V.; LANNA, L.; ROYO, V. de A. Avaliação da qualidade físico-química de comprimidos de enalapril:

referência, genérico e similar, dispensados na rede pública e privada na cidade de Montes Claros-MG

medicines, generic, similar and dispensed by the pharmacy basic containing enalapril. Therefore, we performed quality control physicochemical four samples enalapril tablets sold in pharmacies and dispensed by basic pharmacy located in Montes Claros, MG, Brazil. The samples were evaluated with respect to visual appearance, weight, strength (hardness) and disintegration according to the methodologies described in the Brazilian Pharmacopoeia. The results showed that all product reviews are approved in all tests. Thus, the samples were analyzed in accordance with the official specifications.

Keywords: Enalapril. Quality control. Tablets.

\section{INTRODUÇÃO}

A hipertensão arterial sistêmica (HAS) é uma condição clínica multifatorial, crônica, não transmissível que se caracteriza por níveis elevados e sustentados de pressão arterial (PA) (SOCIEDADE BRASILEIRA DE CARDIOLOGIA, 2010). Trata-se da doença de maior frequência na população brasileira, um dos grandes problemas de saúde pública no Brasil e no mundo (LOTUFO, 2005; ROSARIO et al., 2009; SOCIEDADE BRASILEIRA DE CARDIOLOGIA, 2010) que, devido a sua alta prevalência e baixas taxas de controle, se torna um dos principais fatores de risco de doenças cardiovasculares (DCV) (SOCIEDADE BRASILEIRA DE CARDIOLOGIA, 2010).

A escolha adequada para o início e manutenção do tratamento anti-hipertensivo, medicamentoso ou não, é feita considerando características tanto dos indivíduos quanto dos medicamentos, como: idade, etnia, interações com outros medicamentos, efeitos adversos, contraindicações e custo-efetividade. Os IECA (inibidores da enzima conversora de angiotensina) são geralmente os fármacos de escolha para adultos com menos de 55 anos (ROCHA, 2012).

As drogas da classe IECA possibilitaram grande avanço no tratamento da hipertensão arterial sistêmica. $\mathrm{O}$ desenvolvimento dos primeiros compostos desta classe desencadeou uma enorme expansão no conhecimento do sistema renina-angiotensina. Os IECA inibem a formação de angiotensina II. Podem ser usados isoladamente ou em combinação com outras drogas, apresentam alta eficácia em diminuir a pressão arterial na maioria dos pacientes. Possuem perfis metabólico e hemodinâmicos favoráveis e podem ser usados na vigência de outras doenças associadas. Têm poucos efeitos adversos e excelente tolerabilidade subjetiva (SILVA, 2006). 
CÂMARA, R. P.; BATISTA, T. N. V.; LANNA, L.; ROYO, V. de A. Avaliação da qualidade físico-química de comprimidos de enalapril: referência, genérico e similar, dispensados na rede pública e privada na cidade de Montes Claros-MG

O enalapril é um representante dos IECA derivado de dois aminoácidos, a L-alanina e a L-prolina; uma pró-droga com potencial de ação muito inferior ao metabólito ativo, este precisa ser hidrolisado no fígado para assim produzir ácido dicarboxílico ativo, o enalaprilato (BONAZZI et al., 1997; JACKSON, 2005; LIMA; SANTOS; LIMA, 2008; PORTOLÉS et al., 2004). É muito utilizado pela sua rápida absorção e boa biodisponibilidade (PORTOLÉS et al., 2004).

A existência de dificuldades econômicas da população em geral torna interessante a utilização de medicamentos genéricos e similares, como alternativa ao de referência. Porém, é imprescindível que ambas as especialidades farmacêuticas apresentem eficácia e segurança equivalentes ao medicamento referência para tornar essa troca possível. Isso é importante para que o paciente tenha um tratamento de qualidade com custo inferior, não tendo, assim, qualquer prejuízo em seu tratamento (LINSBINSKI; MUSIS; MACHADO, 2008).

O controle de qualidade, conjunto de medidas destinadas a verificar a qualidade de cada lote de medicamentos, objetiva verificar se o produto está em conformidade com as especificações farmacopéicas; este é um requisito das Boas Práticas de Fabricação (BPF) e é executado em todas as etapas de produção do medicamento, a fim de evitar falhas e detectar desvios da qualidade do produto que possam implicar em riscos ao paciente (RIGOBELLO et al., 2013).

Os comprimidos, formas farmacêuticas de consistência sólida, são obtidos pela compressão de substâncias medicamentosas e excipientes em equipamento adequado, tais como diluentes, lubrificantes, desintegrantes, aglutinantes, corantes e edulcorantes (PEIXOTO et al., 2005). Devem apresentar estabilidade física e química, desintegrar-se no tempo previsto, ser pouco friáveis, apresentar integridade e superfície lisa e brilhante, sendo destituídos de alguns defeitos como falhas, fissuras e contaminação, compatíveis em relação à espessura, diâmetro, tamanho, peso, forma e dureza (ANSEL et al., 2000; BANKER; ANDERSON, 2001; PEIXOTO et al., 2005).

A avaliação da qualidade de comprimidos é necessária para que seja liberado o medicamento para o mercado em condições que garantam sua segurança, eficácia terapêutica e qualidade, durante todo o prazo de validade (PEIXOTO et al., 2005). Os testes físicoquímicos de equivalência farmacêutica podem ser considerados um indicativo da bioequivalência entre medicamentos em teste e referência (STORPIRTIS et al., 2004) e, juntamente com o cumprimento das Boas Práticas de Fabricação e Controle de Qualidade (BPFC), fornecem as bases técnicas e científicas para a intercambialidade entre o genérico e 
CÂMARA, R. P.; BATISTA, T. N. V.; LANNA, L.; ROYO, V. de A. Avaliação da qualidade físico-química de comprimidos de enalapril:

referência, genérico e similar, dispensados na rede pública e privada na cidade de Montes Claros-MG

seu medicamento de referência, pois esses podem ser considerados equivalentes terapêuticos (BENET, 1999; MEREDITH, 1996; MEYER, 1999; STORPIRTIS et al., 2004).

No mercado farmacêutico brasileiro, encontra-se o maleato de enalapril registrado como referência, genérico e similar. Pelas diferenças significativas de custo entre medicamentos genéricos e similares em relação ao medicamento referência, surgem entre consumidores e até profissionais da saúde dúvidas acerca da qualidade e eficácia desses medicamentos. A execução de testes que avaliem a qualidade dos comprimidos de captopril produzidos por diferentes indústrias permite verificar a possibilidade de intercambialidade entre eles e sua conformidade com os requisitos farmacopeicos da monografia individual.

\section{METODOLOGIA}

Realizou-se uma pesquisa experimental, por meio de ensaios físico-químicos para testar a qualidade dos medicamentos em estudo. Foi avaliada a qualidade de quatro lotes de comprimidos de enalapril $20 \mathrm{mg}$ de diferentes laboratórios, sendo estes obtidos pela compra nas apresentações de referência, genérico, similar e obtidos em farmácia da rede básica da cidade de Montes Claros - MG.

Foram adquiridos 90 comprimidos de cada uma das amostras totalizando 360 comprimidos. Os seguintes ensaios físico-químicos foram realizados em triplicata: aspecto visual, peso médio, desintegração e dureza. Os métodos e os parâmetros para a aprovação adotados foram baseados na Farmacopeia Brasileira (2010).

\subsection{Aspecto Visual}

Analisou-se o aspecto visual das amostras quanto à uniformidade de coloração dos comprimidos, comprimidos faltantes, quebrados ou trincados, legibilidade na embalagem e qualquer outra alteração aparente.

\subsection{Peso médio}

O teste de peso médio de comprimidos foi efetuado pela da média aritmética obtida na pesagem individual de 20 unidades, em triplicata, seguindo as especificações farmacopeicas. 
CÂMARA, R. P.; BATISTA, T. N. V.; LANNA, L.; ROYO, V. de A. Avaliação da qualidade físico-química de comprimidos de enalapril: referência, genérico e similar, dispensados na rede pública e privada na cidade de Montes Claros-MG

Pode-se tolerar não mais do que cinco unidades fora dos limites de $\pm 7,5 \%$ em relação ao peso médio, e, nenhuma unidade poderá estar acima ou abaixo de $15 \%$, conforme descrito na Farmacopeia Brasileira (2010).

\subsection{Dureza}

Durante a produção de comprimidos, são realizadas determinações de dureza, a fim de verificar a necessidade de ajustes de pressão nas máquinas de compressão. O teste para determinação da dureza foi realizado por meio de um aparelho denominado durômetro, o qual mediu a força aplicada diametralmente, necessária para esmagar dez comprimidos de enalapril $20 \mathrm{mg}$, individualmente, sendo a força medida em Newton $(\mathrm{N})$ e o valor mínimo aceitável de $30 \mathrm{~N}$, aproximadamente $3 \mathrm{Kgf}$ (FARMACOPÉIA BRASILEIRA, 2010).

\subsection{Desintegração}

O teste de desintegração permite verificar se comprimidos e cápsulas se desintegram dentro do limite de tempo especificado, no caso de comprimidos em 30 minutos, quando seis unidades do lote são submetidas à ação de aparelhagem específica sob condições experimentais descritas.

Foram utilizados seis comprimidos no teste, um em cada um dos seis tubos da cesta, adicionando um disco a cada tubo e imergindo-os no aparelho, utilizando água mantida a $37 \pm$ $1{ }^{\circ} \mathrm{C}$ como líquido de imersão, conforme descrito na Farmacopeia Brasileira (2010).

\section{RESULTADOS E DISCUSSÃO}

A Organização Mundial de Saúde (OMS) propõe que, para o uso racional de medicamentos, é preciso, além de estabelecer a necessidade do uso do medicamento, que este seja apropriado, prescrito adequadamente, na forma farmacêutica, doses e período de duração do tratamento apropriadas, disponível de modo oportuno, a um preço acessível, que se dispense em condições adequadas, com a necessária orientação e responsabilidade. Também preconiza que se cumpra o regime terapêutico já prescrito e que o medicamento responda sempre aos critérios de qualidade exigidos, de acordo com as especificações de eficácia e segurança comprovados e aceitáveis (AQUINO, 2008; BRASIL, 2001). 
CÂMARA, R. P.; BATISTA, T. N. V.; LANNA, L.; ROYO, V. de A. Avaliação da qualidade físico-química de comprimidos de enalapril: referência, genérico e similar, dispensados na rede pública e privada na cidade de Montes Claros-MG

Os desvios da qualidade dos medicamentos trazem inúmeras implicações, faz-se, então, importante o controle de qualidade das formulações farmacêuticas para garantir que os medicamentos tenham suas características repetidas de maneira uniforme em cada lote de produção (LACHMAN; HANNA; LIN, 2001; RIBEIRO, 2012).

Tabela 1 - Resultados dos testes realizados na avaliação da qualidade de comprimidos de enalapril

\begin{tabular}{|c|c|c|c|c|c|c|}
\hline Amostra & Aspecto & & eso Médio & & Desintegração & Dureza * \\
\hline & & gramas & $\pm d p$ & $\pm \%$ & (min) & Newtons \\
\hline Referência A & Conforme & 0,20045 & 0,001431 & 0,71 & & 300,0 \\
\hline Referência B & Conforme & 0,20085 & 0,001236 & 0,61 & 5,00 & 299,7 \\
\hline Referência C & Conforme & 0,20190 & 0,001446 & 0,71 & & 300,0 \\
\hline Genérico A & Conforme & 0,17595 & 0,005424 & 3,08 & & 299,8 \\
\hline Genérico B & Conforme & 0,17865 & 0,004682 & 2,62 & 1,07 & 299,4 \\
\hline Genérico C & Conforme & 0,18075 & 0,003492 & 1,93 & & 299,8 \\
\hline Similar A & Conforme & 0,15975 & 0,002074 & 1,29 & & 299,6 \\
\hline Similar B & Conforme & 0,15930 & 0,001720 & 1,07 & 3,15 & 299,4 \\
\hline Similar C & Conforme & 0,15975 & 0,001372 & 0,85 & & 299,4 \\
\hline Farmácia Básica A & Conforme & 0,16069 & 0,002975 & 1,76 & & 299,9 \\
\hline Farmácia Básica B & Conforme & 0,16915 & 0,001531 & 0,91 & 3,32 & 298,9 \\
\hline Farmácia Básica C & Conforme & 0,16850 & 0,003517 & 2,08 & & 299,8 \\
\hline
\end{tabular}

Nota: *Valor de referência da Dureza: > $30 \mathrm{~N}$

Os comprimidos devem apresentar integridade, superfície homogênea, lisa e brilhante, coloração característica, sendo destituídos de alguns defeitos como falhas, fissuras e contaminação (BANKER; ANDERSON, 2001). Após inspeção das amostras, a partir dos resultados obtidos, constatou-se que todas as amostras analisadas apresentaram-se de acordo com as recomendações descritas acima.

Os comprimidos devem desintegrar-se no tempo previsto, apresentar estabilidade física e química e serem pouco friáveis (BANKER; ANDERSON, 2001). Podem, porém, variar entre si no tamanho, forma, peso, dureza, espessura, características de desintegração e outros aspectos, dependendo do uso a que se destina e do método de fabricação (ANSEL et al., 2000). Esses fatores devem ser controlados durante a produção de comprimidos, a fim de assegurar a aparência do produto e a sua eficácia terapêutica (ANSEL et al., 2000). Dessa forma, as amostras foram submetidas às análises físicas e os resultados obtidos foram comparados com as especificações da Farmacopeia Brasileira (2010) e são apresentados na 
CÂMARA, R. P.; BATISTA, T. N. V.; LANNA, L.; ROYO, V. de A. Avaliação da qualidade físico-química de comprimidos de enalapril: referência, genérico e similar, dispensados na rede pública e privada na cidade de Montes Claros-MG

TAB. 1. Conforme os resultados obtidos, observou-se que as análises de peso médio, dureza e tempo de desintegração apresentaram-se dentro das especificações da literatura consultada.

A partir dos valores obtidos para os pesos individuais encontrados e do cálculo do peso médio, foi determinado o limite de variação. O peso médio (referência: 0,201067 g, genérico: 0,17845 g, similar: 0,1596 g, farmácia basica: 0,16865 g) dos comprimidos de enalapril está situado entre 80,0 e 250,0 mg, sendo, nesse caso, aceitável a variação de não mais do que duas unidades no limite de $\pm 7,5 \%$ e nenhuma unidade com variação de $\pm 15,0 \%$. Nenhum comprimido analisado ficou fora dos limites especificados. Portanto, os valores encontrados estão em conformidade com as especificações estabelecidas pela Farmacopéia Brasileira (2010) em relação ao peso dos comprimidos. Uma vez que as fórmulas estão baseadas no peso das formas farmacêuticas, a determinação e os ajustes dos pesos dos comprimidos, ao longo do processo de compressão, são procedimentos importantes; o peso irá influenciar, também, na concentração de princípios ativos em cada unidade (RIBEIRO, 2012).

O teste de dureza consiste na resistência do comprimido ao esmagamento ou à ruptura sob pressão radial. Esse teste consiste em submeter o comprimido à ação de um aparelho que meça a força aplicada diametralmente, necessária para esmagá-lo. A dureza de um comprimido é proporcional ao logaritmo da força de compressão e inversamente proporcional à sua porosidade. Durante a produção, determinações de dureza são realizadas, a fim de verificar a necessidade de ajustes de pressão na máquina de compressão (FARMACOPEIA BRASILEIRA, 2010). Para verificar a resistência mecânica dos comprimidos de enalapril 20 mg, foi determinada a dureza de dez comprimidos, em Newton (unidade de força). Foi observado que todos os comprimidos foram rompidos com uma força acima de $30 \mathrm{~N}$, que corresponde à força mínima aceitável para que ocorra o rompimento dos comprimidos no teste de dureza, especificado pela Farmacopéia Brasileira (2010). Portanto, os comprimidos analisados apresentaram uma resistência mecânica (referência: 299,9 N, genérico: 299,6 N, similar: 299,5 N, farmácia básica: 299,5 N) adequada às abrasões e aos choques mecânicos durante a produção, transporte, armazenamento, distribuição e manuseio.

Valores elevados para as mesmas medidas podem indicar a ocorrência de descalibração da força exercida pelas punções da máquina de moldagem dos comprimidos. Esta é uma ocorrência normal em equipamentos industriais que são utilizados em larga escala, porém, pode refletir em diferentes velocidades de desintegração dos lotes produzidos, com consequentes variações do perfil de biodisponibilidade entre as unidades. Isso justifica a 
CÂMARA, R. P.; BATISTA, T. N. V.; LANNA, L.; ROYO, V. de A. Avaliação da qualidade físico-química de comprimidos de enalapril: referência, genérico e similar, dispensados na rede pública e privada na cidade de Montes Claros-MG

exigência da legislação de programas de calibração e validação periódicos desses equipamentos (BRASIL, 2003).

A desintegração é definida como o estado no qual nenhum resíduo da unidade (cápsula ou comprimido), salvo fragmentos de revestimento ou matriz de cápsulas insolúveis, permanece na tela metálica do aparelho de desintegração. Esta afeta diretamente a absorção, a biodisponibilidade e a ação terapêutica do fármaco. Para que o princípio ativo fique disponível para ser absorvido e exerça sua ação farmacológica, é necessário que ocorra a desintegração do comprimido em pequenas partículas, aumentando-se a superfície de contato com o meio de dissolução, favorecendo, portanto, a absorção e a biodisponibilidade do fármaco no organismo. A desintegração de comprimidos não deve ser superior a 30 minutos. Diante disso, todas as amostras analisadas neste estudo foram submetidas à determinação do tempo de desintegração. A partir dos resultados obtidos (referência: 5,0 min, genérico: 1,07 min, similar: 3,15 min, farmácia básica: 3,32 min), todas as amostras foram aprovadas nesse teste.

Foram encontrados alguns trabalhos que avaliaram a qualidade de medicamentos contendo enalapril. Bianchin et al. (2012) encontraram uma amostra reprovada no ensaio de friabilidade, ao analisarem duas amostras adquiridas no período de junho de 2007 a julho de 2008 pela Secretaria Municipal de Saúde de uma cidade do Sul do Brasil, avaliando aspecto visual, peso médio, friabilidade, dissolução, doseamento, teor de princípio ativo. Gomes et al. (2005) analisaram 35 produtos acabados (cápsulas contendo 5,10, 20 ou $50 \mathrm{mg}$ de maleato de enalapril) fornecidos por diversas farmácias de manipulação num período de 12 meses. Após o cálculo do teor a partir da análise em HPLC, foi demonstrado que $26 \%$ das amostras dos produtos acabados encontravam-se em desacordo.

\section{CONCLUSÃO}

A qualidade garantida de um produto pode ser entendida como o conjunto de propriedades e características que o tornam satisfatório para uso, atendendo às necessidades dos consumidores. Em vista disso, a avaliação da qualidade de medicamentos na Indústria Farmacêutica representa uma etapa indispensável para a sua comercialização em condições adequadas de uso.

O objetivo deste trabalho consistiu na avaliação da qualidade de comprimidos de enalapril $20 \mathrm{mg}$ dispensados em farmácias comerciais e farmácia básica em Montes Claros 
CÂMARA, R. P.; BATISTA, T. N. V.; LANNA, L.; ROYO, V. de A. Avaliação da qualidade físico-química de comprimidos de enalapril: referência, genérico e similar, dispensados na rede pública e privada na cidade de Montes Claros-MG

(MG), em que se verificou a conformidade dos medicamentos com as especificações farmacopéicas, avaliando, assim, a possibilidade de desvios quanto à qualidade, segurança e eficácia do medicamento.

A partir dos resultados obtidos para os parâmetros investigados neste trabalho, observou-se que todas as amostras estão em concordância com as especificações farmacopéicas, foram aprovadas nos testes gerais de controle de qualidade dos medicamentos em estudo. Portanto, as amostras de comprimidos de enalapril foram qualificadas como produtos adequados para o consumo de acordo com os ensaios realizados.

\section{REFERÊNCIAS}

ANSEL, H. C.; POPOVICH, N. G.; ALLEN, L. Farmacotécnica: formas farmacêuticas e liberação de fármacos. 6. ed. São Paulo: Editorial Premier, 2000.

AQUINO, D. S. de. Por que o uso racional de medicamentos deve ser uma prioridade? Ciência e Saúde Coletiva, Rio de Janeiro, v. 13, supl. abr. 2008.

BANKER, G. S.; ANDERSON, N. R. Comprimidos. In: LACHMAN, L.; LIEBERNAN, H. A.; KANIG, J. L. Teoria e prática na indústria farmacêutica. 7. ed. Lisboa: Fundação Calouste Gulbenkian, 2001. p. 509-526.

BENET, L. Z. Understanding bioequivalence testing. Transplantation Proceedings, New York, v. 31, n. 3, p. 75-95, 1999.

BIANCHIN, M. D. et al. Avaliação da qualidade de comprimidos de propranolol e enalapril distribuídos no sistema público de saúde em uma cidade do sul do Brasil. Ciência e Saúde Coletiva, Rio de Janeiro, v. 17, n. 2, fev. 2012.

BONAZZI, D. et al. Analysis of ACE inhibitors in pharmaceutical dosage forms by derivative UV spectroscopy and liquid chromatography (HPLC). Journal of Pharmaceutical and Biomedical Analysis, v. 16, p. 431-438, 1997.

BRASIL. Ministério da Saúde. Política nacional de medicamentos. Brasília, 2001.

BRASIL. Agência Nacional de Vigilância Sanitária. Resolução da Diretoria Colegiada 135 de 01 de junho de 2003. Regulamento técnico para medicamentos genéricos. Brasília, 2003.

FARMACOPÉIA BRASILEIRA. 5. ed. São Paulo: Atheneu, 2010.

GOMES, T. C. F.; CIRILO, H. N. C.; MIRANDA, C. G.; PAULA, J. R.; BARA, M. T. F. Avaliação do teor de maleato de enalapril em matérias-primas e produtos acabados. Revista Eletrônica de Farmácia, v. 2, n. 2, p. 92-95, 2005. 
CÂMARA, R. P.; BATISTA, T. N. V.; LANNA, L.; ROYO, V. de A. Avaliação da qualidade físico-química de comprimidos de enalapril: referência, genérico e similar, dispensados na rede pública e privada na cidade de Montes Claros-MG

JACKSON, E. K. Renina e Angiotensina. In: GOODMAN, L. S.; GILMAN, A. As bases farmacológicas da terapêutica. 10. ed. Rio de Janeiro: McGraw-Hill, 2005. p. 609-628.

LACHMAN, L.; HANNA, S. A.; LIN, K. Controlo e garantia de qualidade. In: LACHMAN, L.; LIEBERMAN, H. A.; KANIG, J. L. Teoria e prática na indústria farmacêutica. Lisboa: Fundação Calouste Gulbenkian, 2001. v. 2, p. 1357-1439.

LIMA, D. M; SANTOS, L. D; LIMA, E. M. Stability and in vitro release profile of enalapril maleate from different commercially available tablets: possible therapeutic implications.

Journal of Pharmaceutical and Biomedical Analysis, v. 47, p. 934-937, 2008.

LINSBINSKI, L. M.; MUSIS, C. R.; MACHADO, S. R. P. Avaliação da equivalência farmacêutica de comprimidos de captopril. Revista Brasileira de Farmácia, Cuiabá, v. 89, n. 3, p. 214-219, 2008.

LOTUFO, P. A. Stroke in Brazil: a neglected disease. São Paulo Medical Journal, São Paulo, v. 123, n. 1, jan. 2005.

MEREDITH, P. A. Generic drugs: therapeutic equivalence. Drug Safety, Auckland, v. 15, n. 4, p. 233-242, 1996.

MEYER, G. F. History and regulatory issues of generic drugs. Transplantation Proceedings, New York, v. 31, n. 3, p. 105-125, 1999.

PEIXOTO, M. M. et al. Avaliação da qualidade de comprimidos de Captopril dispensados em Feira de Santana-BA. Infarma, São Paulo, v. 16, n. 13-14, p. 69-73, 2005.

PORTOLÉS, A. et al. Bioequivalence study of two formulations of enalapril, at a single oral dose of 20mg (Tablets): a randomized, twoway, open-label, crossover study in healthy volunteers. Current Therapeutic Research, v. 65, p. 34-46, 2004.

RIGOBELLO, C. et al. Avaliação da qualidade e perfil de dissolução de comprimidos de cloridrato de propanolol. Acta Scientiarum, Maringá, SP, v. 35, n. 1, 2013.

ROCHA, E. II Simpósio Luso-Brasileiro de hipertensão terapêutica: opções na abordagem dos subgrupos. Revista Brasileira de Hipertensão, v. 19, n. 4, 2012.

ROSARIO, T. M. et al. Prevalência, controle e tratamento da hipertensão arterial sistêmica em Nobres - MT. Arquivos Brasileiros de Cardiologia, São Paulo, v. 93, n. 6, dez. 2009.

RIBEIRO, P. R. S. Controle de qualidade de medicamentos anti-hipertensivos similares comercializados em farmácias de Imperatriz, MA, Brasil. Cadernos de Pesquisa, v. 19, n. 1. 2012.

SILVA, P. Farmacologia. 7. ed. Rio de Janeiro: Guanabara Koogan, 2006. 
CÂMARA, R. P.; BATISTA, T. N. V.; LANNA, L.; ROYO, V. de A. Avaliação da qualidade físico-química de comprimidos de enalapril: referência, genérico e similar, dispensados na rede pública e privada na cidade de Montes Claros-MG

SOCIEDADE BRASILEIRA DE CARDIOLOGIA / SOCIEDADE BRASILEIRA DE HIPERTENSÃO / SOCIEDADE BRASILEIRA DE NEFROLOGIA. VI Diretrizes Brasileiras de Hipertensão. Arquivos Brasileiros de Cardiologia, São Paulo, v. 95, p. 1-51, 2010.

STORPIRTIS, S. et al. A equivalência farmacêutica no contexto da intercambialidade entre medicamentos genéricos e de referência: bases técnicas e científicas. Infarma, São Paulo, v. 16, n. 10, 2004. 